Int. J. Electrochem. Sci., 14 (2019) 4595 - 4610

International Journal of

ELECTROCHEMICAL

SCIENCE

WWW.electrochemsci.org

\title{
PEDOT: PSS-exfoliated Graphene to Improve the Corrosion Resistance of Waterborne Epoxy Coating
}

\author{
Yue Sul, ${ }^{1,2}$, Shihui Qiu ${ }^{1,2}$, Yu Liu ${ }^{1,}$, Dongping Yang ${ }^{1,}$,Haichao Zhao ${ }^{1, *}$, Liping Wang ${ }^{1, *}$ \\ ${ }^{1}$ Key Laboratory of Marine Materials and Related Technologies, Zhejiang Key Laboratory of Marine \\ Materials and Protective Technologies, Ningbo Institute of Materials Technology and Engineering, \\ Chinese Academy of Sciences, Ningbo 315201, P. R. China \\ ${ }^{2}$ University of Chinese Academy of Sciences, Beijing 100049, China \\ *E-mail: zhaohaichao@nimte.ac.cn, wangliping@nimte.ac.cn
}

doi: $10.20964 / 2019.05 .37$

Received: 2 January 2019 / Accepted: 11 February 2019 / Published: 10 April 2019

In this paper, we prepared PEDOT:PSS-G composites on the basis of $\pi$ - $\pi$ interactions between PEDOT:PSS and graphene. It was revealed that PEDOT:PSS has been inserted into the graphene sheets and graphene sheets were peeled off into 4-5 layers by SPM analysis. Electrochemical impedance spectroscopy and micro-electrochemical spectrum showed the agglomerated graphene not only couldn't act as barriers, but also accelerated the corrosion reaction; while the exfoliated graphene fully exerted its barrier effect and increased the diffusion path for corroded medium. Meanwhile, the addition of PEDOT:PSS could promote the formation of passivation layers on the steel surface, and inhibited the corrosion reaction from further occurring. The two effects complemented each other to improve the corrosion resistance of the coating.

Keywords: Graphene; PEDOT:PSS; Corrosion inhibition; Cooperative effect

\section{FULL TEXT}

(C) 2019 The Authors. Published by ESG (www.electrochemsci.org). This article is an open access article distributed under the terms and conditions of the Creative Commons Attribution license (http://creativecommons.org/licenses/by/4.0/). 